\title{
The Use of QT Prolongation Risk Factor Assessment Tool to Assess the Risk of QT Prolongation in Patients Taking Methadone: An Irish Experience
}

\author{
Maitiu O. Tuathail ${ }^{1,2}$ \\ ${ }^{1}$ Irish College of General Practitioners, Dublin 2, Dublin, Ireland \\ ${ }^{2}$ Ranelagh Medical, Dublin 6, Dublin, Ireland
}

Email address:

maitiuotuathail@rcsi.ie,maitiuotuathail@rcsi.ie

\section{To cite this article:}

Maitiu O. Tuathail, The Use of QT Prolongation Risk Factor Assessment Tool to Assess the Risk of QT Prolongation in Patients Taking Methadone: An Irish Experience. European Journal of Clinical and Biomedical Sciences. Vol. 7, No. 5, 2021, pp. 75-80.

doi: $10.11648 /$ j.ejcbs.20210705.11

Received: May 4, 2021; Accepted: May 17, 2021; Published: September 16, 2021

\begin{abstract}
Currently, no tool exists to assist doctors in estimating their patients' risk of QT prolongation. Methadone is one of many medications which is known to prolong a patient's QT, and which can lead to Torsades de Pointes and sudden cardiac death. The aim of the study was to design a QT prolongation risk factor assessment tool, for patients taking methadone, which would allow physicians to objectively assess a patient's risk of QT prolongation. Following a literature review a QT prolongation risk factor assessment tool was created. An audit was then carried out to see what risk factors for QT prolongation were identified prior to use of the tool, in our practice, and then after the tool had been introduced. The introduction of a dedicated QT risk factor assessment tool led to an 61\% (39/100 to 100/100) increase in the documentation of individual QT prolongation risk factors in our patients. There was a $100 \%(4 / 4)$ increase in the documentation of risk of QT prolongation in the patient notes, of discussion that risk with patients (4/4), and of the frequency of repeat ECGS (4/4). The introduction of such a QT prolongation risk factor assessment tool in our practice led to an increased awareness of QT prolonging medications, an improvement in the documentation of QT prolonging risk factors and a change in the culture of the practice around medication prescribing and QT prolongation. In patients who are on methadone, a QT prolongation risk factor assessment tool allows for an objective assessment of each patient's risk of QT prolongation which then allows for measures to be put in place to monitor and address this risk.
\end{abstract}

Keywords: Methadone, Addiction, QTC Prolongation

\section{Introduction}

Methadone remains the gold standard treatment in opioid replacement therapy [1] with an estimated 650, 000 people currently using methadone worldwide [2]. This accounts for less than $10 \%$ of those who are in need of treatment, with 10,000 patients currently on methadone treatment in Ireland [3].

Methadone is generally considered to be a safe drug, but like all medications, it has side effects, some of which can be fatal, most commonly respiratory depression secondary to overdose [4]. Methadone has also been implicated in sudden cardiac death thought to be due to the development of malignant arrythmias such as torsade's de pointes [4]. Prolongation of the QT interval is the mechanism through which these malignant arrythmias occur, and this can be detected by the use of electrocardiograph (ECG) in patients [4].

Despite this, knowledge of the potential for methadone to prolong the QT interval amongst physicians who prescribed methadone in the United states has been found to be as low as $41 \%$ [5]. Unfortunately, Irish data on this is currently lacking and due to the differences in the structures around addiction services between both nations, the American figures cannot be extrapolated to draw a conclusion on the situation in Ireland.

Currently no consensus exists nationally or internationally, with regards to routine use of ECG in screening for QT prolongation in patients on methadone. The Irish College of General Practitioners / Irish College of Psychiatry / Health 
Service Executive clinical guidelines for opioid substitution treatment acknowledges this lack of uniformity in clinical guidance [6]. That said, these guidelines do outline scenarios where clinicians should consider performing an ECG on patients deemed at risk of QT prolongation [6].

In the UK, the drug misuse and dependence, UK guidelines on clinical management published by Public health England state that in some cases an ECG may be deemed necessary at induction or intermittently on a case by case basis based on the individual patients risk of QT prolongation as "assessed by the prescribing physician", similar to Irish guidelines [4].

Finally, the medicines and healthcare products regulatory agency (MHRA) in the UK outlines scenarios where clinicians should consider performing an ECG, but like the aforementioned guidelines these are suggestions, are not obligatory, and much is left to the prescribing physicians interpretation of the guidelines and subjective clinical opinion of risk [4].

To summarise the problem as it currently stands, the estimation of risk of QT prolongation remains subjective, and currently no objective assessment or assessment tool exists to assist with this.

What complicates this further, in an Irish context, is that many methadone prescribing GPs here exclusively manage the patient's opioid dependency and do not provide routine GP services to their patients, and may therefore not be in a situation to comprehensively assess the patients risk for QT prolongation [3]. Furthermore, many of these patients are under the care of psychiatry services and are also receiving anti-depressant or anti-psychotic medication, both of which independently can prolong the QT [6].

Currently, no guidance or assessment tool exists either within our practice or nationally with regards to the assessment of the risk of QT prolongation in patients on methadone. To address this the author set about designing a QT prolongation risk factor assessment tool. This was done following an extensive literature review on the topics of QT prolongation, and QT prolongation in patients taking methadone.

The objectives of the tool was to provide a method to ensure all patients in the practice had their QT prolongation risk assessed and documented, to ensure that all patients deemed at risk of QT prolongation had an ECG at appropriate intervals, to review the patient's current medication list to ensure they are not on QT prolonging medication, to ensure all patients have annual blood tests to monitor for electrolyte and hormonal abnormalities and finally, to ensure all patients are informed of the risk of QT prolongation. These objectives were decided upon following the literature review.

QT Prolongation Risk factor ossesment

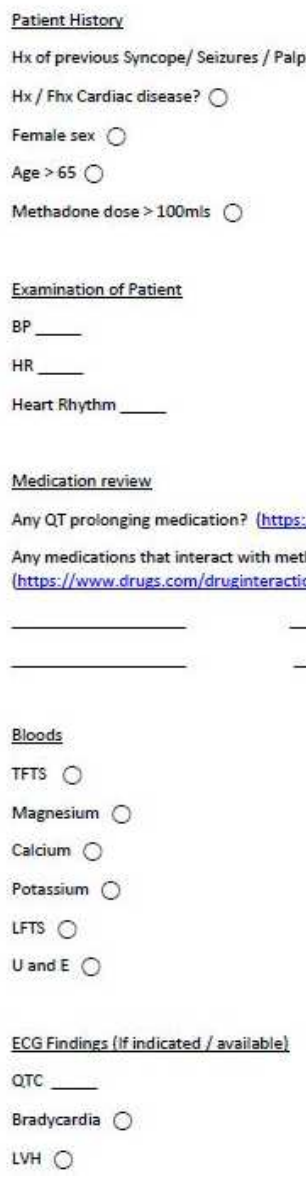

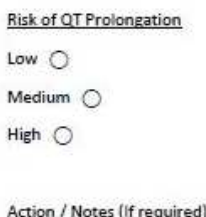

Action / Notes (lif required

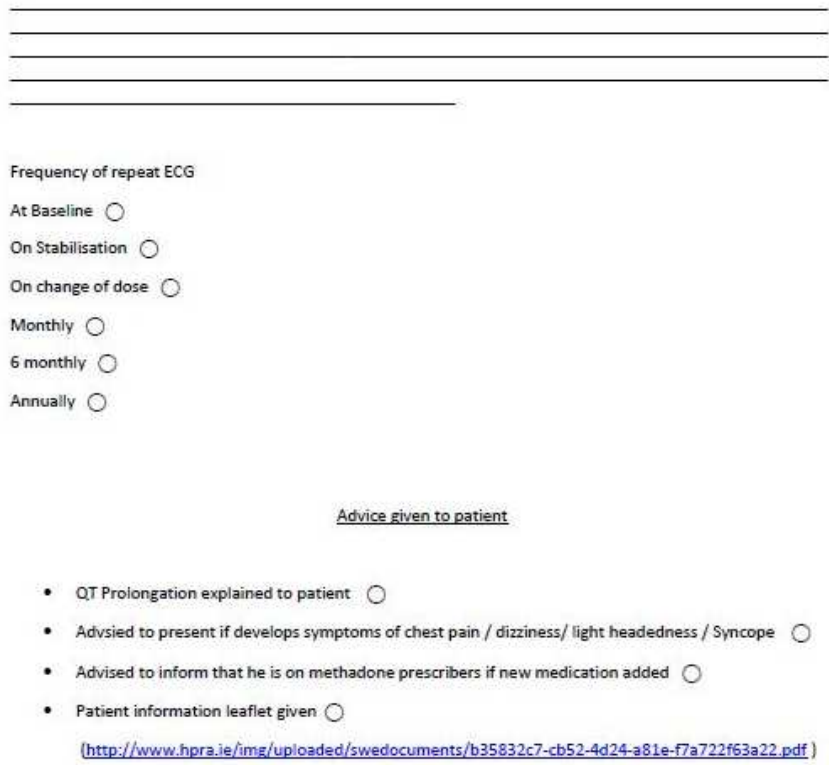

Signed Dr

Date

Figure 1. The QT prolongation risk factor assessment tool. 


\section{Literature Review}

QT prolongation is defined as a QTc interval greater than $450 \mathrm{~mm}$ in men and $470 \mathrm{~mm}$ in women [7], and the use of a simple ECG tracing is sufficient to reliably and accurately diagnose it [8].

The knowledge of QT prolongation and its complex relationship with medications has been known about for over 100 years [9]. There are certain categories of medications, mainly antiarrhythmics and antipsychotics which have formed the bulk of research into this area. This research has evaluated that the risk of QT prolongation leading to torsade de pointes varies hugely between drugs, from a negligible risk to a risk as high as $9 \%$ for the cardiac drug quinidine [7].

The measures taken to identify and mitigate the risk of QT prolongation equally vary hugely. Measures are now in place to monitor this risk in patients on antipsychotic medication where clear guidelines exist for the prescribing clinicians [10]. Furthermore, the associated risk of "metabolic syndrome" and the adverse impact this can have on cardiac disease is also recognized in this patient cohort, and appropriate screening and preventative measures exist to monitor this [11].

In contrast, as described, similar guidelines on QT prolongation and methadone prescribing do not exist. Methadone use is of itself not a risk factor for the development of cardiac disease, nor does it exacerbate existing cardiac disease, but cardiac disease is a risk factor for prolongation of the QT interval and therefore this should also be factored into, and assessed independently of the risk of QT prolongation in patients on methadone [7].

To contextualize this problem, the incidence of QT prolongation in patients on antipsychotic medication has been estimated at $5.7 \%$ [12]. This contrasts sharply to the incidence of QT prolongation in patients on methadone which has been estimated to be between $16 \%$ [13] and $23 \%$ [14] with most having two or more additional risk factors for QT prolongation as well as methadone use [15].

The Irish College of General Practitioners (ICGP) have set out criteria for assessing the risk for QT prolongation [3]. These are patients on a dose over $100 \mathrm{mg}$ of methadone, patients being prescribed other known QT prolonging medicines, patients with a personal or family history of significant cardiac disease, and finally patients experiencing unexplained syncope or generalized seizures.

The medicines and healthcare products regulatory agency (MHRA) in the UK have outlined additional criteria for QT risk assessment [5]. These are patients with electrolyte abnormalities, patients with liver disease and finally patients with cardiovascular disease [5].

Finally, additional risk factors for QT prolongation were identified by a 2011 national medicines information centre bulletin, which included female sex, age $>65$ and bradycardia [16].

There is no consensus as to whether a baseline ECG should be done at induction or how frequently ECGs should be performed on those who are identified as being at risk of QT prolongation [3]. A 2008 national medicines information centre bulletin on drug induced QT interval prolongation suggested that ECG's be done, at baseline, prior to increases in the dose of methadone, after stabilisation on methadone and finally, monthly for the first six months and six monthly thereafter [17].

The guidelines surrounding methadone prescribing and addiction do not comment on monitoring of electrolyte abnormalities. Electrolyte abnormalities such as hypokalaemia, hypocalcaemia and hypomagnesemia increase the risk of QT prolongation as do hormonal imbalances such as hypo and hyperthyroidism [4], and therefore monitoring of electrolyte and hormone levels is prudent in patients on methadone.

Finally, it is considered good practice to inform patients of their risk of QT prolongation [3].

\section{Methods}

Prior to this study no assessment tool existed within our practice or nationally with regards to the assessment of the risk of QT prolongation in patients taking methadone.

As discussed, a QT prolongation risk factor assessment tool was created following an extensive literature review. The effectiveness of the tool was assessed using an audit. The identification and documentation of QT prolonging risk factors was assessed prior to and following the introduction of the QT prolongation risk factor assessment tool.

The objectives of the audit were set out as follows. To ensure all patients in the practice have their QT prolongation risk assessed and documented by $15^{\text {th }}$ March 2020. To ensure that all patients deemed at risk of QT prolongation have an ECG at appropriate intervals. To ensure an alert is set up on the file with regards to the patients QT. To review the patient's current medication list to ensure they are not on QT prolonging medication. To ensure all patients have annual blood tests to monitor for electrolyte and hormonal abnormalities. Finally, to ensure all patients are informed of the risk of QT prolongation.

Data was collected using the practice software, which allowed the identification of the patients in our practice who are on methadone.

The patients note 's were reviewed to see if the practice had already met some objectives prior to the implementation of the practice QT prolongation risk assessment tool. The intervention then occurred, which was introduction of the QT prolongation risk assessment tool.

The audit cycle was then repeated one-month post introduction of the tool to see if the objectives have been met. Full adherence to tool was considered to be the standard for the purposes of the audit, that is a $100 \%$ compliance rate with all the objectives. 


\section{Results}

\subsection{Pre-Intervention}

A search was undertaken using the practice electronic medical record system which identified that the practice had four patients currently taking methadone.

The search of the EMR revealed that the overall QT risk factor identification and documentation rate was $39 \%$ (39/100).

History of relevant risk factors was noted in $12 \%$ of cases (2/16). Demographics were accurately recorded 100\% (4/4) of cases. Vitals were documented in $44 \%(7 / 16)$ of cases.

The patient's current medication was documented (4/4) but not reviewed (0/4).

Blood test results were documented in 58\% (14/24) of cases.

ECG and ECG findings were documented in $25 \%(4 / 16)$ of cases.
Risk of QT Prolongation, frequency of repeat ECGS and dates of next review were not documented in any cases $(0 / 4)$.

\subsection{Post Intervention}

The post intervention results showed that the introduction of a dedicated QT risk factor assessment tool led to an $61 \%$ $(39 / 100$ to $100 / 100)$ increase in the documentation of individual QT prolongation risk factors in our patients.

It also led to a $100 \%$ increase in the documentation of drug interactions with methadone and also of co-prescribing with other QT prescribing medication ( $0 / 4$ to $4 / 4)$.

There was a $100 \%(4 / 4)$ increase in the documentation of risk of QT prolongation in the patient notes, of discussion of the risk of QT prolongation with patients (4/4) and of the frequency of repeat ECGS (4/4). Baseline ECGS with baseline QT intervals have were established and documented on all patients $(4 / 4)$.

Table 1. Pre intervention results.

\begin{tabular}{|c|c|c|}
\hline Documentation & Noted & Not noted \\
\hline Hx of Previous events noted & 1 & 3 \\
\hline Hx of Cardiac disease noted & 1 & 3 \\
\hline Hx of Liver / Renal disease & 0 & 4 \\
\hline Dose $>100 \mathrm{mls}$ Documented & 0 & 4 \\
\hline Female sex noted & 4 & 0 \\
\hline Age $>65$ noted & 4 & 0 \\
\hline BP noted & 3 & 1 \\
\hline Heart Rhythm noted & 2 & 2 \\
\hline Medication review noted & 0 & 4 \\
\hline Medication documented & 4 & 0 \\
\hline TFTs documented & 3 & 1 \\
\hline Magnesium documented & 0 & 4 \\
\hline Calcium documented & 2 & 2 \\
\hline Potassium documented & 3 & 1 \\
\hline LFTS documented & 3 & 1 \\
\hline ECG Findings documented & 1 & 3 \\
\hline QTC & 1 & 3 \\
\hline HR & 1 & 3 \\
\hline LVH & 1 & 3 \\
\hline Risk of QT prolongation noted & 0 & 4 \\
\hline Frequency of repeat ECGs noted & 0 & 4 \\
\hline Date of next review & 0 & 4 \\
\hline Documentation of discussion of risk of QT prolongation noted & 0 & 4 \\
\hline
\end{tabular}

History of relevant risk factors was noted in $100 \%$ of cases (16/16). Demographics were accurately recorded 100\% (4/4) of cases. Vitals were documented in $44 \%(7 / 16)$ of cases.

The patient's current medication was documented (4/4) and reviewed (4/4) in all cases.

Blood test results were documented in $100 \%(24 / 24)$ of cases.
ECG and ECG findings were documented in $100 \%(16 / 16)$ of cases.

Risk of QT Prolongation, frequency of repeat ECGS and dates of next review were documented in all cases (4/4).

Overall, the introduction of the QT Prolongation risk factor assessment tool led to the identification and documentation of QT prolonging risk factors in all cases 100\% (100/100).

Table 2. Post intervention results.

\begin{tabular}{lll}
\hline Documentation & Noted & Not noted \\
\hline Hx of Previous events noted & 4 & 0 \\
Hx of Cardiac disease noted & 4 & 0 \\
Hx of Liver / Renal disease & 4 & 0 \\
Dose $>100$ mls documented & 4 & 0 \\
\hline
\end{tabular}




\begin{tabular}{lll}
\hline Documentation & Noted & Not noted \\
\hline Female sex noted & 4 & 0 \\
Age $>$ 65 noted & 4 & 0 \\
BP noted & 4 & 0 \\
HR Noted & 4 & 0 \\
Heart Rhythm noted & 4 & 0 \\
Medication review noted & 4 & 0 \\
Medication documented & 4 & 0 \\
TFTs documented & 4 & 0 \\
Magnesium documented & 4 & 0 \\
Calcium documented & 4 & 0 \\
Potassium documented & 4 & 0 \\
LFTS documented & 4 & 0 \\
U and E documented & 4 & 0 \\
ECG Findings documented & 4 & 0 \\
QTC & 4 & 0 \\
HR & 4 & 0 \\
LVH & 4 & 0 \\
Risk of QT prolongation noted & 4 & 0 \\
Frequency of repeat ECGs noted & 4 & 0 \\
Date of next review & 4 & 0 \\
Documentation of discussion of risk of QT prolongation noted & 4 & \\
\hline
\end{tabular}

\subsection{Aims and Objectives}

As outlined in table 3, the implementation of the practice's QT prolongation risk factor assessment tool allowed the practice to achieve both the aims and objectives as outlined fully.

Table 3. Objectives achieved.

\begin{tabular}{ll}
\hline Objective & Achieved \\
\hline To ensure all patients in the practice have their QT prolongation risk assessed and documented by $15^{\text {th }}$ march 2020 \\
To ensure that all patients deemed at risk of QT prolongation have an ECG at appropriate intervals. \\
$\begin{array}{ll}\text { To ensure an alert is set up on the file with regards to the patients QT } & \text { Yes } \\
\text { To review the patient's current medication list to ensure they are not on QT prolonging medication } & \text { Yes } \\
\text { To ensure all patients have annual blood tests to monitor for electrolyte and hormonal abnormalities. To ensure all patients are informed } & \text { Yes } \\
\text { of the risk of QT prolongation. } & \text { Yes }\end{array}$ \\
\hline
\end{tabular}

\section{Discussion}

The introduction of the QT prolongation risk factor assessment tool showed a dramatic improvement in the practice's documentation of QT prolonging risk factors.

It also led to an increased awareness of the QT prolonging effects of medication and in particular methadone amongst the clinical staff in the practice. This will undoubtedly have beneficial effects on patient outcomes, with reduced prescribing of medications that can prolong the QT interval when prescribed with methadone, but also importantly, medications that can interact with methadone and lead to increase in methadone plasma concentrations.

Furthermore, what the introduction of the tool has done is led to a change in the culture within the practice around medication prescribing, and the importance of QT prolongation and possible drug interactions, in that process. This has had benefits beyond methadone prescribing and the QT prolongation risk factor assessment tool.

It was difficult to compare this audit to other studies, as other tools to assess the risk of QT prolongation in patients on methadone do not exist. There is a lack of guidance in this area, and guidelines, both here and the UK do make some recommendations, but ultimately the decision on how and when to assess for QT prolongation lies with the prescribing clinician. This ambiguity is most certainly down to the fact that research into this area is lacking.

For our methadone patients, the QT prolongation risk factor assessment tool has allowed for a structed review of QT risk at every interaction with these patients. Prior to this, a subjective estimation was undertaken by doctors on an ad-hoc basis, with no documentation of this. What the dedicated QT prolongation risk factor assessment tool has done if facilitate a change in culture within the practice, one where a more objective rather than a subjective estimation of risk is undertaken coupled with a formal documentation of this.

Given the litigious and increasingly medicolegal environment that clinicians are operating in, combined with the ever-increasing complexities of our patients' comorbidities, I believe erring on the side of caution is important, with clear documentation of risk, in conjunction with clear evidence of actions taken to mitigate that risk.

Given the small sample size the author recommends that the QT risk factor prolongation tool be studied in a larger population cohort. The author also recommends that a scoring system be incorporated into the tool to allow a robust, numerical assessment of the risk of QT prolongation for patients on methadone. 


\section{Conclusion}

No tool exist in Ireland to assist clinicians who prescribe methadone in objectively assessing a patients risk of QT prolongation.

The introduction of such a QT prolongation risk factor assessment tool in our practice led to an increased awareness of QT prolonging medications, an improvement in the documentation of QT prolonging risk factors and a change in the culture of the practice around medication prescribing and QT prolongation.

The author believes that the introduction of a QT Prolongation risk factor assessment tool allows clinicians to assess the risk of QT prolongation in their patients in an informed, workable and sustainable manner, and importantly, allows for the clear documentation of both this, and the steps taken to address this risk.

\section{References}

[1] Pp P, Trogu E, Maremmani I, Pacini M. QTc interval screening for cardiac risk in methadone treatment of opioid dependence - Pani - 2013 - Cochrane Database of Systematic Reviews - Wiley Online Library. 2013; (6).

[2] WHO. No Title. Bull world Heal Organ. 2008; 86 (3): $161-$ 240 .

[3] Delargy I, Crowley D, Van Hout MC. Twenty years of the methadone treatment protocol in Ireland: reflections on the role of general practice. Harm Reduct J. 2019; 16 (1).

[4] England P health,. Drug Misuse and Dependence. Drug Misuse Depend Uk Guid Clin Manag. 2017; 68-9.

[5] Krantz MJ, Rowan SB, Schmittner J, Bucher Bartelson B. Physician awareness of the cardiac effects of methadone: results of a national survey. J Addict Dis. 2007; 26 (4): 79-85.

[6] Health Service Executive. Clinical Guidelines for Opioid Substitution Treatment. Heal Serv Exec [Internet]. 2017; Available http://hse.ie/eng/services/publications/Primary/clinicalguidelines-for-opioid-substitution-treatment.pdf
[7] Taylor DM. Antipsychotics and QT prolongation. Acta Psychiatr Scand. 2003; 107 (2): 85-95.

[8] Noel ZR, See VY, Flannery AH. Walk the Line-The Importance of Well-Informed Interpretation of QT Prolongation. Ann Pharmacother. 2021; 55 (1): 123-6.

[9] Schwartz PJ, Woosley RL, Woosley RL. Predicting the Unpredictable: Drug-Induced QT Prolongation and Torsades de Pointes. J Am Coll Cardiol. 2016; 67 (13): 1639-50.

[10] Sussex Partnership NHS. Guidance on the Use of Antipsychotics version 3.2. 2015; (October 2015): 1-61. Available from: https://www.sussexpartnership.nhs.uk/sites/default/files/docu ments/antipsychotic_guidelines_version_3.2_-_oct 15__with_new_hdat_form_added_-_dec_16_0.pdf

[11] Cooper SJ, Reynolds GP, Barnes TRE, England E, Haddad PM, Heald A, et al. BAP guidelines on the management of weight gain, metabolic disturbances and cardiovascular risk associated with psychosis and antipsychotic drug treatment. J Psychopharmacol. 2016; 30 (8): 717-48.

[12] Ali Z, Ismail M, Nazar Z, Khan F, Khan Q, Noor S. Prevalence of QTc interval prolongation and its associated risk factors among psychiatric patients: a prospective observational study. BMC Psychiatry [Internet]. 2020; 20 (1): 277. Available from: https://doi.org/10.1186/s12888-020-02687-w

[13] Ogungbe O, Akil L, Ahmad HA. Exploring unconventional riskfactors for cardiovascular diseases: Has opioid therapy been overlooked? Int J Environ Res Public Health. 2019; 16 (14).

[14] Krantz MJ, Martin J, Stimmel B, Mehta D, Haigney MCP. QTc interval screening in methadone treatment. Ann Intern Med. 2009; 150 (6): 387-95.

[15] Jennifer M Treece, Mohammad Al Madani, George El Khoury, Ola Khraisha, James E Martin, Steven J Baumrucker, Christopher A Neglia TKP. Comprehensive Review on Methadone-Induced QT Prolongation and Torsades. J Pharmacol Pharmacother. 2016; 2 (2): 3-7.

[16] St James' Hospital, National Medicines Information Centre. 2011; 17 (4): 11-2.

[17] St James' Hospital, National Medicines Information Centre. 2008; 14 (5). 